\title{
RIEMANN-LEBESGUE PROPERTIES OF BANACH SPACES ASSOCIATED WITH SUBSETS OF COUNTABLE DISCRETE ABELIAN GROUPS
}

\author{
PATRICK N. DOWLING and NARCISSE RANDRIANANTOANINA \\ Department of Mathematics and Statistics, Miami University, Oxford, OH 45056 \\ e-mail:dowlinpn@muohio.edu,e-mail:randrin@muohio.edu
}

(Received 5 December, 2001; accepted 17 May, 2002)

\begin{abstract}
For a subset $\Lambda$ of the dual group of a compact metrizable abelian group, we introduce the type I-, II-, and III- $\Lambda$-Riemann-Lebesgue property of a Banach space. As an application we use these properties to characterize Rajchman sets.
\end{abstract}

2000 Mathematics Subject Classification. 46B22, 46S10, 43A25.

1. Introduction. Generalizations of the Radon-Nikodym property and the analytic Radon-Nikodym property of Banach spaces have been extensively studied over the past twenty years. Edgar [7] and later Dowling [6], introduced and studied Radon-Nikodym properties associated with subsets of countable discrete abelian groups (types I, II and III- $\Lambda$-RNP). Robdera and Saab [11] introduced and studied the concept of the analytic complete continuity property and later introduced complete continuity properties associated with subsets of countable discrete abelian groups (types I, II and III- $\Lambda$-CCP) [12]. Of particular interest is that $L^{1}[0,1]$ has type I, II or III- $\Lambda$-RNP (or CCP) if and only if $\Lambda$ is a Riesz set.

In a recent paper, Bu and Chill [2] introduced the notions of the Riemann-Lebesgue property and the analytic Riemann-Lebesgue property, which are weakenings of the complete continuity property and the analytic complete continuity property, respectively. In this note, we shall define and study Riemann-Lebesgue properties of Banach spaces associated with subsets of countable discrete abelian groups. In particular, we shall give conditions under which $L^{1}[0,1]$ has a Riemann-Lebesgue property.

2. Preliminaries and definitions. Throughout this paper, $G$ will denote a compact metrizable abelian group, $\mathcal{B}(G)$ is the $\sigma$-algebra of Borel subsets of $G$, and $\lambda$ is normalized Haar measure on $G$. The dual group of $G$ will be denoted by $\Gamma$. We note that $\Gamma$ is a countable discrete abelian group [13].

Let $X$ be a complex Banach space and let $1 \leq p \leq \infty$. For an $X$-valued measure, $\mu$ on $\mathcal{B}(G)$ we define

$$
\mathbb{E}(\mu \mid \pi)=\sum_{E \in \pi} \frac{\mu(E)}{\lambda(E)} \chi_{E},
$$

The second author was supported in part by NSF Grant DMS-0096696. 
where $\pi$ is a finite measurable partition of $G$, along with the convention $\frac{0}{0}=0$. The space $V^{p}(G ; X)$ consists of all $X$-valued measures $\mu$ on $\mathcal{B}(G)$ with $\|\mu\|_{p}<\infty$, where

$$
\|\mu\|_{p}=\sup _{\pi}\|\mathbb{E}(\mu \mid \pi)\|_{L^{p}(G ; X)}
$$

and the supremum is taken over all finite measurable partitions of $G$.

If $\mu \in V^{P}(G ; X)$ and $\gamma \in \Gamma$, then the Fourier coefficient $\hat{\mu}(\gamma)$ is defined by

$$
\hat{\mu}(\gamma)=\int_{G} \bar{\gamma}(x) d \mu(x) .
$$

If $f \in L^{p}(G ; X)$ and $\gamma \in \Gamma$, then $\hat{f}(\gamma)$ is defined by

$$
\hat{f}(\gamma)=\int_{G} f(x) \bar{\gamma}(x) d \lambda(x) .
$$

If $\Lambda \subseteq \Gamma$, we define

$$
L_{\Lambda}^{p}(G ; X)=\left\{f \in L^{p}(G ; X): \hat{f}(\gamma)=0 \text { for all } \gamma \notin \Lambda\right\}
$$

and

$$
V_{\Lambda}^{p}(G ; X)=\left\{\mu \in V^{p}(G ; X): \hat{\mu}(\gamma)=0 \text { for all } \gamma \notin \Lambda\right\}
$$

We also need the following notation:

$V_{\Lambda, a c}^{1}(G ; X)=\left\{\mu \in V^{1}(G ; X): \mu\right.$ is $\lambda$ - continuous and $\hat{\mu}(\gamma)=0$ for all $\left.\gamma \notin \Lambda\right\}$.

For more information on vector measures, we refer the reader to the monograph of Diestel and Uhl [3]. We are now ready to define Riemann-Lebesgue properties associated to a subset $\Lambda$ of $\Gamma$. To motivate these definitions recall that a Banach space $X$ has type-I- $\Lambda$-Radon-Nikodym property (I- $\Lambda$-RNP), (resp. type II- $\Lambda$-RadonNikodym property (II- $\Lambda$-RNP)) if every $\mu \in V_{\Lambda}^{\infty}(G ; X)$ (resp. every $\mu \in V_{\Lambda, a c}^{1}(G ; X)$ ) is differentiable [5], and $X$ has the type-I- $\Lambda$-complete continuity property (I- $\Lambda$-CCP), (resp. type-II- $\Lambda$-complete continuity property (II- $\Lambda$-CCP)) if every $\mu \in V_{\Lambda}^{\infty}(G ; X)$ (resp. every $\mu \in V_{\Lambda, a c}^{1}(G ; X)$ ) has relatively compact range [12].

Definition 1. Let $G$ be a compact abelian metrizable group and let $\Lambda$ be a subset of $\Gamma$. A Banach space $X$ is said to have the type-I- $\Lambda$-Riemann-Lebesgue property (I- $\Lambda$-RLP) if every $\mu \in V_{\Lambda}^{\infty}(G ; X)$ satisfies $\lim _{\substack{\gamma \rightarrow \infty \\ \gamma \in \Lambda}}\|\hat{\mu}(\gamma)\|=0$.

REMARK. It is easily seen that I- $\Lambda$-RNP implies I- $\Lambda$-CCP and I- $\Lambda$-CCP implies I- $\Lambda$-RLP.

Definition 2. Let $G$ be a compact abelian metrizable group and let $\Lambda$ be a subset of $\Gamma$. A Banach space $X$ is said to have the type-II- $\Lambda$-Riemann-Lebesgue property (II- $\Lambda$-RLP) if every $\mu \in V_{\Lambda, a c}^{1}(G ; X)$ satisfies $\lim _{\substack{\gamma \rightarrow \infty \\ \gamma \in \Lambda}}\|\hat{\mu}(\gamma)\|=0$.

REMARK. It is obvious that II- $\Lambda$-RLP implies I- $\Lambda$-RLP, since every element of $V_{\Lambda}^{\infty}(G ; X)$ is an element of $V_{\Lambda, a c}^{1}(G ; X)$. Also II- $\Lambda$-RNP implies II- $\Lambda$-CCP and II- $\Lambda$ CCP implies II- $\Lambda$-RLP. Furthermore, by the method of proof of [12, Theorem 4.3] we can see that I- $\Gamma-\mathrm{RLP}$ is equivalent to II- $\Gamma$-RLP. 
REMARK. If $G=\mathbb{T}$, the circle group, then $\Gamma=\mathbb{Z}$. In [2], Bu and Chill defined the notion of a Banach space having the Riemann-Lebesgue property. It can be easily seen that their concept of Riemann-Lebesgue property is equivalent to both I-ZZ-RLP and II-ZZ-RLP. The concept of the analytic Riemann-Lebesgue property introduced by $\mathrm{Bu}$ and Chill is equivalent to both I-(N $\cup\{0\})$-RLP and II-( $\mathbb{N} \cup\{0\})$-RLP.

REMARK. It is clear that if a Banach space has I- $\Lambda$-RLP (resp. II- $\Lambda$-RLP), then so does every subspace of $X$. Moreover, since $G$ is compact and metrizable, $\mathcal{B}(G)$ is countably generated and hence every element of $V_{\Lambda, a c}^{1}(G, X)$ has separable range. Consequently, $X$ has I- $\Lambda$-RLP (resp. II- $\Lambda$-RLP) if every separable subspace of $X$ has I- $\Lambda$-RLP (resp. II- $\Lambda$-RLP).

To motivate the third type of Riemann-Lebesgue property we recall that a Banach space $X$ has type-III- $\Lambda$-Radon-Nikodym property (III- $\Lambda$-RNP), (resp. type III- $\Lambda$ complete continuity property (III- $\Lambda$-CCP)) if every absolutely summing operator $T$ : $C(G) \rightarrow X$ with $T \equiv 0$ on $C_{\Lambda^{\prime}}(G)$ is nuclear (resp. compact).

DEFINITION 3. Let $G$ be a compact abelian metrizable group and let $\Lambda$ be a subset of $\Gamma$. A Banach space $X$ is said to have type-III- $\Lambda$-Riemann-Lebesgue property (III- $\Lambda$ RLP) if every absolutely summing operator $T: C(G) \rightarrow X$ with $T \equiv 0$ on $C_{\Lambda^{\prime}}(G)$ has the property that $\{T(\bar{\gamma}): \gamma \in \Lambda\}$ is relatively compact in $X$.

REMARK. It is clear that III- $\Lambda$-RNP implies III- $\Lambda$-CCP and III- $\Lambda$-CCP implies III- $\Lambda$-RLP. It is not so obvious that III- $\Lambda$-RLP implies II- $\Lambda$-RLP. This is the result we shall now prove.

Proposition 4. Let $G$ be a compact abelian metrizable group and let $\Lambda \subseteq \Gamma$. If a Banach space $X$ has III- $\Lambda-R L P$, then $X$ has $I I-\Lambda-R L P$.

Proof. Suppose that $X$ has III- $\Lambda$-RLP and let $\mu \in V_{\Lambda, a c}^{1}(G ; X)$. Define an operator $T: C(G) \rightarrow X$ by

$$
T(f)=\int_{G} f d \mu \quad \text { for all } f \in C(G) .
$$

Since $\mu$ is the representing measure for $T$ and $\mu$ is of bounded variation, $T$ is an absolutely summing operator. Also, if $\gamma \in \Lambda^{\prime}$, then $\bar{\gamma} \notin \Lambda$ and so $T(\gamma)=\int_{G} \gamma d \mu=$ $\hat{\mu}(\bar{\gamma})=0$. Hence $T \equiv 0$. on $C_{\Lambda^{\prime}}(G)$. Therefore, since $X$ has III- $\Lambda$-RLP, $\{T(\bar{\gamma}): \gamma \in \Lambda\}$ is relatively norm compact in $X$. This means that $\{\hat{\mu}(\gamma): \gamma \in \Lambda\}$ is relatively norm compact in $X$. For each $x^{*} \in X^{*}, x^{*} \mu$ is a scalar measure of bounded variation that is absolutely continuous with respect to $\lambda$. Hence $\left.\lim _{\substack{\gamma \rightarrow \infty \\ \gamma \in \Lambda}} x^{*}(\hat{\mu}(\gamma))=\lim _{\substack{\gamma \rightarrow \infty \\ \gamma \in \Lambda}} \widehat{\left(x^{*} \mu\right.}\right)(\gamma)=0$ for all $x^{*} \in X^{*}$. Therefore $(\hat{\mu}(\gamma))_{\gamma \in \Lambda}$ is a weakly null sequence in $X$ and thus, since $\{\hat{\mu}(\gamma): \gamma \in \Lambda\}$ is relatively compact, $(\hat{\mu}(\gamma))_{\gamma \in \Lambda}$ is norm null. This proves that $X$ has II- $\Lambda$-RLP.

3. The results. We begin this section with a characterization of type I- $\Lambda$-RLP. For this result we need to recall the concept of a good approximate identity.

A sequence $\left(i_{n}\right)_{n \in \mathbb{N}}$ of measurable functions $i_{n}: G \rightarrow \mathbb{R}$ is called a good approximate identity on $G$ if

1. $i_{n} \geq 0$ for all $n \in \mathbb{N}$,

2. $\int_{G} i_{n}(x) d \lambda(x)=1$ for all $n \in \mathbb{N}$, 
3. $\sum_{\gamma \in \Gamma} \hat{i}_{n}(\gamma)<\infty$ for all $n \in \mathbb{N}$,

4. $\lim _{n \rightarrow \infty} \int_{U} i_{n}(x) d \lambda(x)=1$ for all neighborhoods $U$ of 1 in $G$.

It is well known that a good approximate identity always exists on $G$ if $G$ is a compact abelian metrizable group.

Our first result is analogous to the Theorem in [7] and Theorem 3.3 in [12]. We shall omit the easy proof.

THEOREM 5. Let $G$ be a compact abelian metrizable group, let $\Lambda \subseteq \Gamma$ and let $\left(i_{n}\right)_{n \in \mathbb{N}}$ be a good approximate identity on $G$. For a Banach space $X$ the following are equivalent.

1. X has $I-\Lambda-R L P$.

2. For each bounded linear operator $S: L^{1}(G) / L_{\Lambda^{\prime}}^{1}(G) \rightarrow X$, the sequence $(S Q(\bar{\gamma}))_{\gamma \in \Lambda}$ is norm null in $X$, where $Q: L^{1}(G) \rightarrow L^{1}(G) / L_{\Lambda^{\prime}}^{1}(G)$ is the natural quotient mapping.

3. If $\left(a_{\gamma}\right)_{\gamma \in \Lambda}$ is a sequence in $X$ such that $\left(f_{n}\right)_{n \in \mathbb{N}}$ is bounded in $L_{\Lambda}^{\infty}(G ; X)$, where $f_{n}=\sum_{\gamma \in \Lambda} \hat{i}_{n}(\gamma) a_{\gamma} \gamma$, then $\left(a_{\gamma}\right)_{\gamma \in \Lambda}$ is a norm null sequence in $X$.

Corollary 6. Let $G$ be a compact abelian metrizable group and let $\Lambda$ be an infinite subset of $\Gamma$. Then $c_{0}$ fails to have $I-\Lambda-R L P$.

Proof. Since $\Lambda$ is countably infinite, $c_{0}$ and $c_{0}(\Lambda)$ are isometric. Let $\left(e_{\gamma}\right)_{\gamma \in \Lambda}$ be the canonical unit vector basis of $c_{0}(\Lambda)$. For each $n \in \mathbb{N}$, define $f_{n}=\sum_{\gamma \in \Lambda} \hat{i}_{n}(\gamma) e_{\gamma} \gamma$. It is easy to see that $\left(f_{n}\right)_{n \in \mathbb{N}}$ is bounded in $L_{\Lambda}^{\infty}\left(G ; c_{0}(\Lambda)\right)$, but $\left(e_{\gamma}\right)_{\gamma \in \Lambda}$ is not a norm null sequence in $c_{0}(\Lambda)$. Thus, by Theorem $5, c_{0}(\Lambda)$ (and hence $c_{0}$ ) fails to have I- $\Lambda$ RLP.

COROLlaRY 7. Let $G$ be a compact abelian metrizable group and let $\Lambda$ be an infinite subset of $\Gamma$. Then $L^{1}(G) / L_{\Lambda^{\prime}}^{1}(G)$ fails to have $I-\Lambda-R L P$.

Proof. Let $Q: L^{1}(G) \rightarrow L^{1}(G) / L_{\Lambda^{\prime}}^{1}(G)$ be the natural quotient mapping and note that $(Q(\bar{\gamma}))_{\gamma \in \Lambda}$ is a sequence of elements of $L^{1}(G) / L_{\Lambda^{\prime}}^{1}(G)$, each with norm at least 1 . Hence, by Theorem $5, L^{1}(G) / L_{\Lambda^{\prime}}^{1}(G)$ fails to have I- $\Lambda$-RLP.

Before we get to our next result, recall that a subset $\Lambda$ of $\Gamma$ is called a Sidon set if $C_{\Lambda}(G)=\ell^{1}(\Lambda)$.

COROLlary 8. Let $G$ be a compact abelian metrizable group, let $\Lambda$ be an infinite Sidon subset of $\Gamma$ and let $X$ be a Banach space. Then $X$ has $I-\Lambda-R L P$ if and only if $X$ does not contain a subspace isomorphic to $c_{0}$.

Proof. Since $\Lambda$ is infinite, every Banach space containing $c_{0}$ will fail to have I- $\Lambda$ RLP, by Corollary 6 . On the other hand, since $\Lambda$ is a Sidon set, every Banach space not containing a subspace isomorphic to $c_{0}$ has the I- $\Lambda$-RNP, by [6], and hence has I- $\Lambda$-RLP.

DEFINITION 9. [9] Let $G$ be a compact abelian metrizable group and let $\Lambda$ be a subset of $\Gamma$. Then $\Lambda$ is called a Rajchman set if each $v \in V_{\Lambda}^{1}(G)$ satisfies $\lim _{\substack{\gamma \rightarrow \infty \\ \gamma \in \Lambda}} \hat{v}(\gamma)=0$.

THEOREM 10. Let $\Lambda$ be a subset of $\Gamma$. Then $\Lambda$ is a Rajchman set if and only if $L^{1}[0,1]$ has $I-\Lambda-R L P$.

Proof. Suppose that $L^{1}[0,1]$ has type I- $\Lambda$-RLP. Since $G$ is compact and metrizable, $L^{1}(G)$ is isomorphic to a subspace of $L^{1}[0,1]$ and hence $L^{1}(G)$ also has type I- $\Lambda$-RLP. 
Let $\mu \in V_{\Lambda}^{1}(G)$. Define $T: L^{1}(G) \rightarrow L^{1}(G)$ by

$$
T h(y)=\overline{(\bar{h} * \mu)(y)}=\overline{\int_{G} \overline{h\left(y x^{-1}\right)} d \mu(x),} \quad \text { for all } h \in L^{1}(G) .
$$

The operator $T$ is clearly linear and is bounded because $\|T h\|_{1} \leq\|h\|_{1}\|\mu\|_{1}$, for all $h \in$ $L^{1}(G)$. Also note that for each $\gamma \in \Gamma, T(\bar{\gamma})=\overline{\hat{\mu}(\gamma) \gamma}$. Therefore, since $\mu \in V_{\Lambda}^{1}(G)$, $T(\bar{\gamma})=0$, for all $\gamma \notin \Lambda$. Hence $\left.T\right|_{L_{\Lambda^{\prime}}^{1}}(G)=0$, and so there exists a bounded linear mapping $S: L^{1}(G) / L_{\Lambda^{\prime}}^{1}(G) \rightarrow L^{1}(G)$ such that $T=S Q$, where $Q: L^{1}(G) \rightarrow L^{1}(G) /$ $L_{\Lambda^{\prime}}^{1}(G)$ is the natural quotient mapping. Hence, by Theorem $5,(T(\bar{\gamma}))_{\gamma \in \Lambda}$ is norm

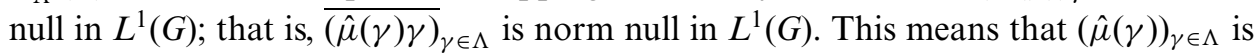
a null sequence in $\mathbb{C}$. Hence $\Lambda$ is a Rajchman set.

Conversely, suppose that $\Lambda$ is a Rajchman set. Let $S: L^{1}(G) / L_{\Lambda^{\prime}}^{1}(G) \rightarrow L^{1}[0,1]$ be a bounded linear operator and define $T: L^{1}(G) \rightarrow L^{1}[0,1]$ by $T=S Q$. By the Fakhoury-Kalton representation theorem $[\mathbf{8}, \mathbf{1 0}]$, there is a family $\left\{\mu_{\omega}\right\}_{\omega \in[0,1]}$ of complex measures $\mu_{\omega}$ on $\mathcal{B}(G)$ such that, for each $f \in L^{1}(G)$, we have

$$
T(f)(\omega)=\int_{G} f(x) d \mu_{\omega}(x),
$$

for $m$-almost all $\omega \in[0,1]$, where $m$ is Lebesgue measure on [0, 1]. The FakhouryKalton representation theorem also says that $\int_{[0,1]}\left\|\mu_{\omega}\right\|_{1} d m(\omega) \leq\|T\|$ and so $\mu_{\omega}$ is of finite variation, for $m$-almost all $\omega \in[0,1]$.

If $\gamma \notin \Lambda$, then $\bar{\gamma} \in \Lambda^{\prime}$ and so $T(\bar{\gamma})=S Q(\bar{\gamma})=0$. Hence for $m$-almost all $\omega \in[0,1]$, we have

$$
0=T(\bar{\gamma})(\omega)=\int_{G} \bar{\gamma}(x) d \mu_{\omega}(x)=\widehat{\mu_{\omega}}(\gamma) .
$$

Since $\Lambda$ is countable we can conclude that, for $m$-almost all $\omega \in[0,1]$, we have $\widehat{\mu_{\omega}}(\gamma)=0$ for all $\gamma \notin \Lambda$. Hence, for $m$-almost all $\omega \in[0,1], \mu_{\omega} \in V_{\Lambda}^{1}(G)$. Therefore, since $\Lambda$ is a Rajchman set, $\left(\widehat{\mu_{\omega}}(\gamma)\right)_{\gamma \in \Lambda}$ is a null sequence in $\mathbb{C}$, for $m$-almost all $\omega \in[0,1]$. Consequently, we have by Lebesgue's dominated convergence theorem

$$
\begin{aligned}
\lim _{\substack{\gamma \rightarrow \infty \\
\gamma \in \Lambda}}\|T(\bar{\gamma})\|_{1} & =\lim _{\substack{\gamma \rightarrow \infty \\
\gamma \in \Lambda}} \int_{[0,1]}|T(\bar{\gamma})(\omega)| d m(\omega) \\
& =\lim _{\substack{\gamma \rightarrow \infty \\
\gamma \in \Lambda}} \int_{[0,1]}\left|\int_{G} \bar{\gamma}(x) d \mu_{\omega}(x)\right| d m(\omega) \\
& =\lim _{\substack{\gamma \rightarrow \infty \\
\gamma \in \Lambda}} \int_{[0,1]}\left|\widehat{\mu_{\omega}}(\gamma)\right| d m(\omega) \\
& =0,
\end{aligned}
$$

because the sequence of functions $\left\{\widehat{\mu_{(\cdot)}}(\gamma)\right\}_{\gamma \in \Lambda}$ converges $m$-almost everywhere to 0 on $[0,1],\left|\widehat{\mu_{(\cdot)}}(\gamma)\right| \leq\left\|\widehat{\mu_{(\cdot)}}\right\|_{1}$, for all $\gamma \in \Lambda$, and $\left\|\widehat{\mu_{(\cdot)}}\right\|_{1}$ is a $m$-integrable function. Thus, by Theorem $5, L^{1}[0,1]$ has type I- $\Lambda$-RLP.

REMARK. Theorem 10 should be compared with the Proposition in [7], where it is proven that $\Lambda$ is a Risez set if and only if $L^{1}[0,1]$ has $\mathrm{I}-\Lambda-\operatorname{RNP}$ ( $\Lambda$ of $\Gamma$ is called a Riesz set if $V_{\Lambda}^{1}(G)=L_{\Lambda}^{1}(G)$ ). It is clear from the definitions of Rajchman and Riesz 
sets, that Riesz sets are Rajchman sets. It is unknown whether Rajchman sets are, in general Riesz sets. We mention also that a characterization of the $\Lambda$ 's for which $L^{1}[0,1]$ has $\mathrm{I}-\Lambda$-CCP is unknown.

We shall now give a characterization of II- $\Lambda$-RLP. This characterization is analogous to Theorem 6 of [5] and Theorem 3.4 of [12]. We omit the easy proof.

THEOREM 11. Let $G$ be a compact abelian metrizable group, let $\Lambda$ be a Riesz subset of $\Gamma$, and let $\left(i_{n}\right)_{n \in \mathbb{N}}$ be a good approximate identity on $G$. For a Banach space $X$ the following are equivalent.

1. X has $I I-\Lambda-R L P$.

2. If $\left(a_{\gamma}\right)_{\gamma \in \Lambda}$ is a sequence in $X$ such that $\left(f_{n}\right)_{n \in \mathbb{N}}$ is bounded in $L_{\Lambda}^{1}(G ; X)$, where $f_{n}=\sum_{\gamma \in \Lambda} \hat{i}_{n}(\gamma) a_{\gamma} \gamma$, then $\left(a_{\gamma}\right)_{\gamma \in \Lambda}$ is a norm null sequence in $X$.

REMARK. One particularly interesting result proved in [2, Proposition 3.7] is that Banach spaces that are B-convex have the Riemann-Lebesgue property. The proof of [2, Proposition 3.7] uses the fact that the Hausdorff-Young inequality holds in Bconvex Banach spaces (see [1] and [4, p. 281]). Using Theorems 5 and 11, we can easily prove that B-convex Banach spaces have II- $\Gamma-$ RLP and therefore have II- $\Lambda$-RLP and I- $\Lambda$-RLP, for all $\Lambda \subseteq \Gamma$.

When $\Lambda$ is a Riesz set, II- $\Lambda$-RNP and III- $\Lambda$-RNP are equivalent [5, Theorem 11], and II- $\Lambda-\mathrm{CCP}$ and III- $\Lambda-\mathrm{CCP}$ are equivalent [12, Proposition 3.8]. We now prove a corresponding result for Riemann-Lebesgue properties.

Proposition 12. Let $G$ be a compact abelian metrizable group, let $\Lambda$ be a Riesz subset of $\Gamma$. Then a Banach space $X$ has $I I-\Lambda-R L P$ if and only if $X$ has III- $\Lambda-R L P$.

Proof. If $X$ has III- $\Lambda$-RLP, then $X$ has II- $\Lambda$-RLP, by Proposition 4.

Conversely, suppose that $X$ has II- $\Lambda$-RLP. Let $T: C(G) \rightarrow X$ be an absolutely summing operator with $T \equiv 0$ on $C_{\Lambda^{\prime}}(G)$. Let $F: \mathcal{B}(G) \rightarrow X^{* *}$ be the representing measure for $T$; that is, $T(f)=\int f d F$ for all $f \in C(G)$. Since $T$ is absolutely summing $F$ is an $X$-valued measure of bounded variation [3]. It is easy to see that $\hat{F}(\gamma)=$ $T(\bar{\gamma})$, for all $\gamma \in \Gamma$. Hence, since $T \equiv 0$ on $C_{\Lambda^{\prime}}(G), \hat{F}(\gamma)=0$, for all $\gamma \notin \Lambda$. Thus $F \in V_{\Lambda}^{1}(G, X)$ and, since $\Lambda$ is a Riesz set, $F \in V_{\Lambda, a c}^{1}(G, X)$. Therefore, since $X$ has II$\Lambda$-RLP, $\lim _{\gamma \rightarrow \infty}\|\hat{F}(\gamma)\|=0$. Consequently, $\lim _{\gamma \rightarrow \infty}\|T(\bar{\gamma})\|=0$ and so $\{T(\bar{\gamma}): \gamma \in \Lambda\}$ is relatively compact in $X$. This proves that $X$ has III- $\Lambda$-RLP.

REMARK. If $\Lambda$ is not a Riesz set, then III- $\Lambda$-RNP is equivalent to the RadonNikodym property and III- $\Lambda$-CCP is equivalent to the complete continuity property. We do not know if there is a corresponding result for Riemann-Lebesgue properties.

THEOREM 13. Let $G$ be a compact abelian metrizable group, let $\Lambda$ be a subset of $\Gamma$. If $X$ is a Banach space such that $L^{1}([0,1], X)$ has $I-\Lambda-R L P$, then $X$ has $I I-\Lambda-R L P$. On the other hand, if $\Lambda$ is a Riesz set, then $X$ has $I I-\Lambda-R L P$ if and only if $L^{1}([0,1], X)$ has $I I-\Lambda-R L P$.

Proof. Suppose that $L^{1}([0,1], X)$ has I- $\Lambda$-RLP. Then $L^{1}(G, X)$ has I- $\Lambda$-RLP. Let $\mu \in V_{\Lambda, a c}^{1}(G, X)$. Define an operator $T: L^{1}(G) \rightarrow L^{1}(G, X)$, by $T(f)=\overline{\mu * \bar{f}}$, for all 
$f \in L^{1}(G)$. Note that for $\gamma \in \Gamma$ and $y \in G$,

$$
\begin{aligned}
(T \gamma)(y) & =\overline{\int \overline{\gamma\left(x^{-1} y\right)} d \mu(x)} \\
& =\overline{\int \gamma(x) \overline{\gamma(y)} d \mu(x)} \\
& =\gamma(y) \overline{\int \gamma(x) d \mu(x)} \\
& =\gamma(y) \overline{\hat{\mu}(\bar{\gamma})} .
\end{aligned}
$$

That is, $T(\gamma)=\overline{\hat{\mu}(\bar{\gamma})} \gamma$, for all $\gamma \in \Gamma$. In particular, if $\gamma \in \Lambda^{\prime}$, then $\bar{\gamma} \notin \Lambda$ so that $T(\gamma)=$ $\overline{\hat{\mu}(\bar{\gamma})} \gamma=0$, since $\mu \in V_{\Lambda, a c}^{1}(G, X)$. Therefore $T \equiv 0$ on $L_{\Lambda^{\prime}}^{1}(G)$ and so there exists an operator $S: L^{1}(G) / L_{\Lambda^{\prime}}^{1}(G) \rightarrow X$ such that $T=S Q$, where $Q: L^{1}(G) \rightarrow L^{1}(G) / L_{\Lambda^{\prime}}^{1}(G)$ is the natural quotient mapping. Hence, by Theorem 5 , since $L^{1}(G, X)$ has I- $\Lambda$-RLP, $\lim _{\substack{\gamma \rightarrow \infty \\ \gamma \in \Lambda}}\|T(\bar{\gamma})\|=0$. That is, $\lim _{\substack{\gamma \rightarrow \infty \\ \gamma \in \Lambda}}\|\hat{\mu}(\gamma)\|=0$. This proves that $X$ has II- $\Lambda$-RLP.

Now suppose that $\Lambda$ is a Riesz set and that $X$ has the II- $\Lambda$-RLP property. Let $T: C(G) \rightarrow L^{1}([0,1], X)$ be an absolutely summing operator with $T \equiv 0$ on $C_{\Lambda^{\prime}}(G)$. By the Fakhoury-Kalton representation theorem $[\mathbf{8}, \mathbf{1 0}]$, there is a family $\left\{\mu_{\omega}\right\}_{\omega \in[0,1]}$ of $X$-valued measures $\mu_{\omega}$ on $\mathcal{B}(G)$, such that, for each $f \in C(G)$, we have

$$
T(f)(\omega)=\int_{G} f(x) d \mu_{\omega}(x), \text { for } m \text {-almost all } \omega \in[0,1] .
$$

Also $\int_{[0,1]}\left\|\mu_{\omega}\right\|_{1} d \lambda(\omega) \leq \pi_{1}(T)$ and so $\mu_{\omega}$ is of finite variation for $m$-almost all $\omega \in$ $[0,1]$.

If $\gamma \notin \Lambda$, then $\bar{\gamma} \in \Lambda^{\prime}$ so $T(\bar{\gamma})=S Q(\bar{\gamma})=0$. Hence for $m$-almost all $\omega \in G$, we have

$$
0=T(\bar{\gamma})(\omega)=\int_{G} \bar{\gamma}(x) d \mu_{\omega}(x)=\widehat{\mu_{\omega}}(\gamma) .
$$

Since $\Lambda$ is countable we can conclude that, for $m$-almost all $\omega \in[0,1]$, we have $\widehat{\mu_{\omega}}(\gamma)=$ 0 , for all $\gamma \notin \Lambda$. Hence, for $m$-almost all $\omega \in G, \mu_{\omega} \in V_{\Lambda}^{1}(G, X)$. Therefore, since $\Lambda$ is a Riesz set, $\mu_{\omega} \in V_{\Lambda, a c}^{1}(G, X)$, for $m$-almost all $\omega \in G$. Since $X$ has II- $\Lambda$-RLP, $\left(\widehat{\mu_{\omega}}(\gamma)\right)_{\gamma \in \Lambda}$ is a null sequence in $X$, for $m$-almost all $\omega \in G$. Finally, applying Lebesgue's dominated convergence theorem, just as in Theorem 10, we get

$$
\begin{aligned}
\lim _{\substack{\gamma \rightarrow \infty \\
\gamma \in \Lambda}}\|T(\bar{\gamma})\|_{L^{1}([0,1], X)} & =\lim _{\substack{\gamma \rightarrow \infty \\
\gamma \in \Lambda}} \int_{[0,1]}\|T(\bar{\gamma})(\omega)\|_{X} d m(\omega) \\
& =\lim _{\substack{\gamma \rightarrow \infty \\
\gamma \in \Lambda}} \int_{[0,1]}\left\|\int_{G} \bar{\gamma}(x) d \mu_{\omega}(x)\right\|_{X} d m(\omega) \\
& =\lim _{\substack{\gamma \rightarrow \infty \\
\gamma \in \Lambda}} \int_{[0,1]}\left\|\widehat{\mu_{\omega}}(\gamma)\right\|_{X} d m(\omega) \\
& =0 .
\end{aligned}
$$

This, in particular, says that $\{T(\bar{\gamma}): \gamma \in \Lambda\}$ is relatively compact in $L^{1}([0,1], X)$. Therefore $L^{1}([0,1], X)$ has III- $\Lambda$-RLP and so it also has II- $\Lambda$-RLP. This completes the proof. 
REMARK. In the proof of Theorem 13, we used the fact that $\Lambda$ is a Riesz set for the sole purpose of concluding that the measures $\mu_{\omega}$ are absolutely continuous. We can drop the Riesz set condition on $\Lambda$ if we replace $L^{1}([0,1], X)$ by $L^{p}([0,1], X)$, where $1<p<\infty$. Specifically, the proof of Theorem 13 can be modified to show that, for any subset $\Lambda$ of $\Gamma$, a Banach space $X$ has III- $\Lambda$-RLP if and only if $L^{p}([0,1], X)$ has III- $\Lambda$-RLP, where $1<p<\infty$.

THEOREM 14. Let $\Lambda$ be a subset of $\Gamma$. Then $\Lambda$ is a Rajchman set if and only if $L^{1}([0,1]$ has $I I I-\Lambda-R L P$.

Proof. If $L^{1}([0,1]$ has III- $\Lambda$-RLP, it has I- $\Lambda$-RLP and so $\Lambda$ is a Rajchman set, by Theorem 10.

For the converse, suppose that $\Lambda$ is a Rajchman set and let $T: C(G) \rightarrow L^{1}([0,1]$ be an absolutely summing operator with $T \equiv 0$ on $C_{\Lambda^{\prime}}(G)$. By the Fakhoury-Kalton representation theorem $[\mathbf{8}, \mathbf{1 0}]$, there is a family $\left\{\mu_{\omega}\right\}_{\omega \in[0,1]}$ of complex measures $\mu_{\omega}$ on $\mathcal{B}(G)$ such that, for each $f \in C(G)$, we have

$$
T(f)(\omega)=\int_{G} f(x) d \mu_{\omega}(x), \quad \text { for almost all } \omega \in[0,1],
$$

and $\int_{[0,1]}\left\|\mu_{\omega}\right\|_{1} d m(\omega) \leq \pi_{1}(T)$, so that $\mu_{\omega}$ is of finite variation, for $m$-almost all $\omega \in$ $[0,1]$. The remainder of the proof is completed just as in the proof of Theorem 10.

\section{REFERENCES}

1. J. Bourgain, A Hausdorff-Young inequality for B-convex Banach spaces, Pacific J. Math. 101 (1982), 255-262.

2. Shangquan $\mathrm{Bu}$ and Ralph Chill, Banach spaces with the Riemann-Lebesgue or the analytic Riemann-Lebesgue property, Bull. London Math. Soc. 34 (2002), 569-581.

3. J. Diestel and J. J. Uhl, Jr., Vector measures (American Mathematical Society, Providence, R.I., 1977). With a foreword by B. J. Pettis, Mathematical Surveys, No. 15.

4. Joe Diestel, Hans Jarchow, and Andrew Tonge, Absolutely summing operators (Cambridge University Press, 1995).

5. Patrick N. Dowling, Radon-Nikodým properties associated with subsets of countable discrete abelian groups, Trans. Amer. Math. Soc. 327 (1991), no. 2, 879-890.

6. Patrick N. Dowling, Duality in some vector-valued function spaces, Rocky Mountain J. Math. 22 (1992), no. 2, 511-518.

7. G. A. Edgar, Banach spaces with the analytic Radon-Nikodým property and compact abelian groups in Almost everywhere convergence (Columbus, OH, 1988), (Academic Press, 1989) 195-213.

8. Hicham Fakhoury, Représentations d'opérateurs à valeurs dans $L^{1}(X, \sigma, \mu)$, Math. Ann. 240 (1979), 203-212.

9. Bernard Host and François Parreau, Ensembles de Rajchman et ensembles de continuité, C. R. Acad. Sci. Paris Sér. A-B 288 (1979), no. 19, A899-A902.

10. N. J. Kalton, Isomorphisms between $L_{p}$-function spaces when $p<1$, J. Funct. Anal. 42 (1981), no. 3, 299-337.

11. Mangatiana A. Robdera and Paulette Saab, The analytic complete continuity property, J. Math. Anal. Appl. 252 (2000), no. 2, 967-979.

12. Mangatiana A. Robdera and Paulette Saab, Complete continuity properties of Banach spaces associated with subsets of a discrete abelian group, Glasgow Math. J. 43 (2001), 185-198.

13. Walter-Rudin, Fourier analysis on groups (Interscience Publishers, 1962). 